\title{
Cuantificación de la eficiencia de la fachada cerámica ventilada mediante técnicas de la mecánica de fluidos computacional
}

\author{
M. MORA PÉREZ ${ }^{1}$, G. LÓPEZ PATIÑO ${ }^{1}$, M. A. BENGOCHEA ESCRIBANO²， P. A. LÓPEZ JIMÉNEZ ${ }^{1}$ \\ 1. Departamento de Ingeniería Hidráulica y Medio Ambiente. Universidad Politécnica de Valencia. \\ 2. KERABEN GRUPO. SA.
}

\begin{abstract}
En muchos países, el calentamiento en los edificios que se da en verano se convierte en una fuente de consumo energético importante. La fachada ventilada se convierte en una herramienta de diseño sostenible, especialmente en diseños preocupados por el bioclimatismo. La fachada ventilada es un sistema de envolvente del edificio basada en la combinación de los paneles cerámicos con la cámara de aire con objeto de favorecer un ahorro energético en condiciones adecuadas, especialmente frente a las necesidades de refrigeración.

El objetivo de la presente contribución es cuantificar la contribución a la eficiencia energética en el comportamiento térmico de los edificios cuando se instala este tipo de fachada. Estas mejoras se deben a la convección producida por el movimiento del aire en la capa de la fachada. El movimiento convectivo del aire está ligado al intercambio de calor con los paneles de la fachada. Esta cuantificación se realiza por medio de la Fluido Dinámica Computacional utilizando un código comercial: STAR CCM+. La metodología propuesta permite una estimación del potencial ahorro de uso de fachada ventilada en unas ciertas condiciones, en relación con el enfriamiento del edificio.
\end{abstract}

Palabras clave: Fachada cerámica ventilada, Fluidodinámica Computacional.

Quantification of ventilated façade efficiency by using computational fluid mechanics techniques

In some countries, summer over-heating is a big problem in a building's energy balance. Ventilated façades are a useful tool when applied to building design, especially in bioclimatic building design. A ventilated facade is a complex, multi-layer structural solution that enables dry installation of the covering elements.

The objective of this paper is to quantify the efficiency improvement in the building thermal when this sort of facade is installed. These improvements are due to convection produced in the air gap of the façade. This convection depends on the air movement inside the gap and the heat transmission in this motion. These quantities are mathematically modelled by Computational Fluid Dynamics (CFD) techniques using a commercial code: STAR CCM+. The proposed method allows an assessment of the energy potential of the ventilated façade and its capacity for cooling.

Keywords: Ceramic Ventilated Façade, Computational Fluid Dynamics.

\section{INTRODUCCIÓN}

\subsection{La fachada cerámica ventilada}

La situación energética actual, enfocada cada vez más a estrategias de sostenibilidad, anima a ingenieros y arquitectos a proponer estrategias constructivas que aprovechen los recursos disponibles y disminuyan el consumo energético en la edificación para mejorar el rendimiento energético de los edificios. En este sentido, una de las estrategias más prometedoras es la fachada ventilada cerámica. Este tipo de fachada tiene un papel destacado en las técnicas de aprovechamiento de las condiciones de la envolvente del edificio muy favorable, y teniendo en cuenta los principios de la ventilación convectiva, tiene grandes posibilidades en el futuro de las estrategias de sostenibilidad en las instalaciones aplicadas a la edificación.

La fachada ventilada es un sistema complejo de estructura multicapa que, sobre el cerramiento de los edificios, permite la instalación de un panel cerámico externo suficientemente

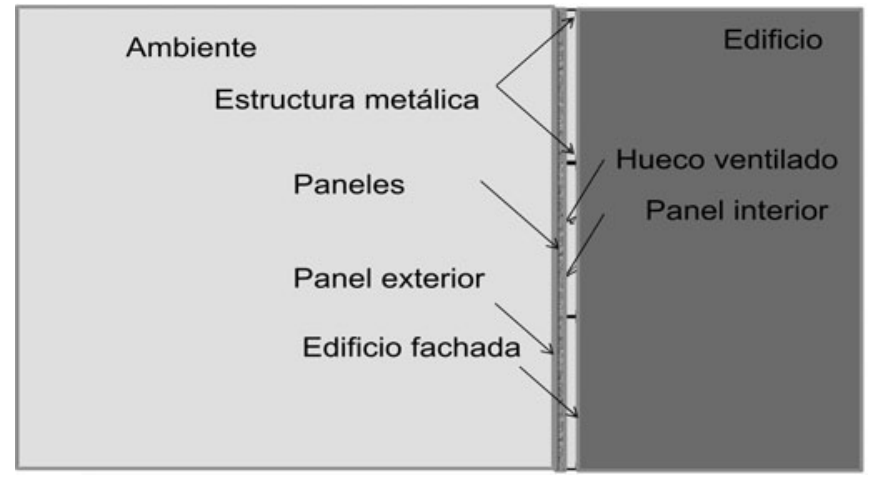

Figura 1. Perfil de la estructura de fachada ventilada con paneles cerámicos 
alejado de dicho cerramiento, de manera que permite el paso de aire entre el panel y el muro (recubierto con una capa de material aislante), con el objeto principal de retirar calor del volumen en cuestión (Figura 1). Es una herramienta muy útil en su aplicación, sobre todo, a la denominada arquitectura bioclimática.

Sin embargo, y a pesar de que la utilidad de la fachada ventilada de paneles cerámicos se conoce empíricamente, la cuantificación del efecto que tiene en el edificio todavía se encuentra en fase de desarrollo. La presente contribución propone una estrategia de parametrización de la eficiencia de la fachada analizando la variación de temperatura que se produce en el espacio ocupado por el aire entre el panel cerámico y el edificio, cuantificando esta variación de temperatura en forma de disminución de potencia calorífica para la refrigeración del volumen interior de las habitaciones del edificio en contacto con la fachada.

Las fachadas cerámicas ventiladas fueron concebidas para proteger los edificios contra una acción combinada de lluvia y viento, aprovechando las ventajas estéticas y de enfriamiento por convección en el flujo dentro de la capa de aire que provee la fachada. Las diferentes capas de materiales que se encuentran en la misma serán:

1. Paneles de material cerámico colocados externamente: esta capa define la apariencia estética del edificio. Desde el punto de vista estructural y estético juega un papel fundamental, pero también afecta a la convección del aire en el interior, puesto que la transmisividad térmica que posea, tendrá influencia sobre la temperatura y calor transmitido al flujo de aire en su interior.

2. Capa de aire. El espesor de esta capa se aconseja sea como mínimo de $3 \mathrm{~cm}$ (según la indicación de salubridad del Código Técnico de la Edificación), con un máximo de 10 $\mathrm{cm}$. El rol que juega esta capa es permitir la circulación de un flujo de aire para eliminar humedades y evacuar calor.

3. Sub-estructura de aluminio. Esta estructura interna típicamente de aluminio está pensada para soportar el material cerámico separado de la pared a la distancia adecuada y darle estabilidad estructural.

Estos materiales, adecuadamente colocados en la fachada ventilada, además de las posibilidades estéticas, deben conseguir mejoras en las condiciones principalmente de verano, pero también de invierno. (Balocco, [2002]).

Especialmente, cuando se considera el papel de enfriamiento de la cara del edificio en contacto con la cámara de aire en la fachada ventilada, hay que analizar el proceso de movimiento del aire en el interior de dicha cámara como consecuencia del efecto convectivo que la misma juega en su dinámica. Este movimiento finalmente va a favorecer el enfriamiento y la energía que se utiliza para el mismo no va a ser transmitida al edificio. Para analizar este movimiento con detalle dentro de la citada cámara de aire, se requiere de un conocimiento de las ecuaciones que rigen la mecánica del flujo, teniendo en cuenta las características del aire como fluido, con un tratamiento adecuado. Para ello, se utilizan técnicas de la mecánica de fluidos computacional (por sus siglas en inglés CFD, Computational Fluid Dynamics) que, resolviendo las ecuaciones del movimiento y conservación de la energía dentro de la cámara de aire, nos permiten cuantificar, simular, visualizar y representar lo fenómenos acaecidos en la región de interés. Las técnicas de modelación mediante CFD han sido ampliamente utilizadas en las últimas décadas para resolución de las ecuaciones del movimiento de fluidos en diversos medios mediante métodos numéricos con gran éxito, como se contrasta en Linden, [1999]; El Sadi y otros, [2010]; Todorovic y otros [2007].

\section{MODELO MATEMÁTICO DEL MOVIMIENTO DE AIRE EN LA CÀMARA DE AIRE DE FACHADA VENTI- LADA CON PANEL CERÁMICO}

\subsection{Geometría para la modelación}

Con el modelo matemático desarrollado se desea conocer el movimiento del aire en la cámara de aire en la fachada ventilada y la evolución de temperaturas en la misma. Para ello se ha considerado una pequeña estructura de un edificio en el cual se simula una cámara de aire de $40 \mathrm{~mm}$ de espesor. En ella se van a aplicar las ecuaciones del movimiento del flujo correspondientes. Para ello se considera una fachada de $6 \mathrm{~m}$ de ancho por $7 \mathrm{~m}$ de alto en una pequeña edificación exenta, puesto que se desea conocer el efecto del aire que penetra en la cámara de la fachada directamente de frente a la misma.
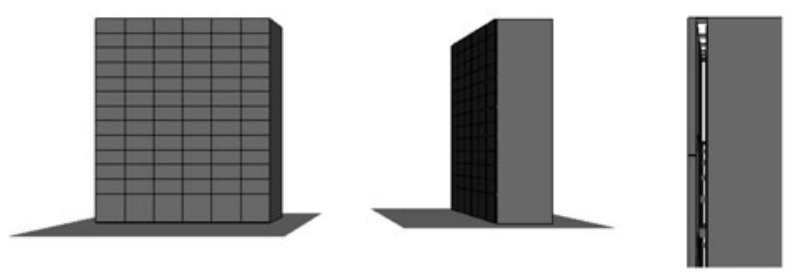

Figura 2a. Fachada esquemática considerada para la modelación del aire en la cámara ventilada

El detalle del aire en la cámara ventilada del que se desea simular el movimiento es el que se muestra en la Figura $2 b$, con una holgura de aire de $40 \mathrm{~mm}$ en esta modelación particular.
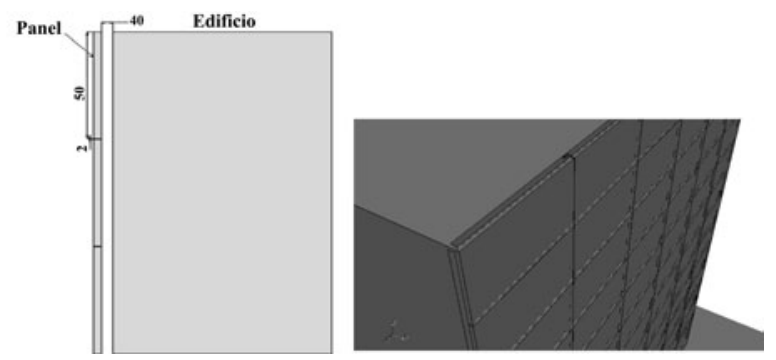

Figura 2b. Detalle de la geometría para el modelo CFD del aire en la fachada

\subsection{Modelo matemático para la fachada cerámica ventilada.}

La mecánica de fluidos computacional (CFD) ha sido ampliamente usada desde los años 70 para la simulación del movimiento del aire tanto dentro de los edificios como en la relación del aire interior con la envolvente exterior. Sin embargo, esta técnica no se ha utilizado para la modelación 
de la fachada ventilada. En la presente contribución se postula como una potente herramienta para cuantificar la capacidad que el flujo convectivo que se crea en la cámara de aire tiene para favorecer el ahorro energético en climatización de los edificios.

Para realizar la representación de cómo las temperaturas varían en el aire que se desplaza por el hueco de la fachada ventilada, se propone el uso del programa comercial para la modelación del movimiento de fluidos STARCCM+. El presente código resuelve numéricamente las ecuaciones de la mecánica de fluidos en un dominio determinado, con unas condiciones de contorno exteriores, aplicadas sobre un volumen que contiene al flujo y en el que se realiza un mallado para llegar a la convergencia de las ecuaciones y la consecución de los perfiles de temperaturas y velocidades en la región de estudio. El uso de estas técnicas computacionales para presentar una visión del comportamiento de los campos de velocidades y presiones; y se presenta como una potente herramienta en el futuro.

El código numérico que se ha utilizado para resolver las ecuaciones de la mecánica de fluidos en el seno de la lámina de aire es el de volúmenes finitos (por sus siglas en inglés FVM), base teórica de todos los programas CFD que existen en el mercado, bien sean comerciales o de libre distribución. El método de los volúmenes finitos permite discretizar y resolver numéricamente ecuaciones diferenciales en un volumen dado. Se considera una malla de discretización del espacio del fluido a modelar. En torno a cada punto de esta malla se construye un volumen de control que no se solapa con los de los puntos vecinos. De esta forma el volumen resultante es igual a la suma de los volúmenes de control considerados. La ecuación diferencial a resolver se integra sobre cada volumen de control, lo cual entrega como resultado una versión discretizada de dicha ecuación, que se resuelve numéricamente.

El modo de proceder en esta modelación es considerar que el flujo total que entra o sale de una de las celdas de la malla a través de sus contornos se conserva. Esta conservación implica que el flujo neto a través de las entradas y salidas es único, a no ser que tengamos fuentes o sumideros en el interior. De hecho, el modelo resuelve ecuaciones de balance en cada una de las celdas, mostrando así solución para las complejas ecuaciones diferenciales que representan al flujo. Sin embargo, en este código en particular, en cada punto se asocia un valor medio de la propiedad calculada, integrando la ecuación diferencial que lo representa. Así, este procedimiento crea un conjunto de ecuaciones lineales cuyos coeficientes son dispersos. De esta forma, se resuelve un sistema de ecuaciones para obtener la distribución de una propiedad en todos los nodos. En este caso, las velocidades, presiones y temperaturas son evaluadas, junto con otros valores derivados de la modelación, principalmente aquellos que se relacionan con la turbulencia.

La ventaja de utilizar estos modelos reside en el hecho de que serán capaces de reproducir problemas reales de la mecánica de fluidos que son imposibles de conocer de otra forma. De hecho, permiten visualizar aspectos hidrodinámicos difíciles de medir o representar en casos reales (por ejemplo líneas de corriente) que suelen tener gran importancia en la comprensión del fenómeno a estudiar.

Como condiciones genéricas sobre el modelo cabe indicar que se ha utilizado, como se ha descrito, el modelo de volúmenes finitos sobre las ecuaciones de la fluidodinámica en la malla que se define mediante el código comercial. Se ha

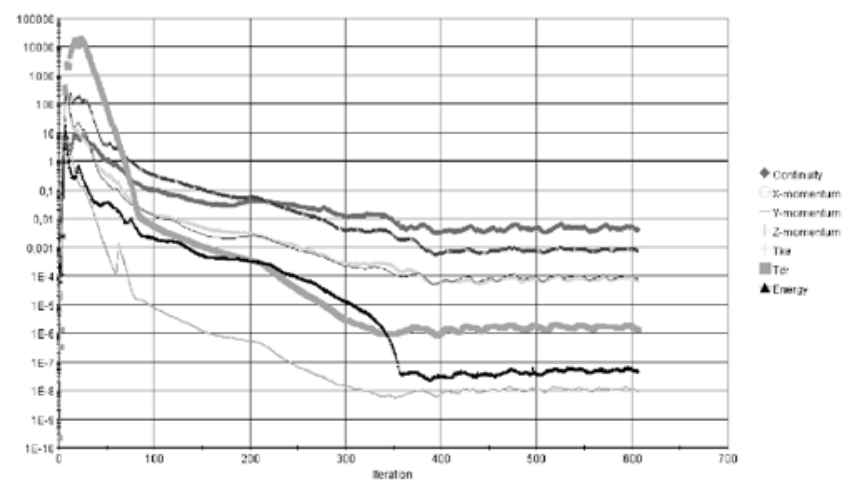

Figura 3. Convergencia de los residuos de la modelación mediante el código utilizado

utilizado un método convencional k-ع para la turbulencia que presenta una convergencia adecuada con un número de 600 iteraciones.

En este caso se ha considerado adecuada la aproximación de Boussinesq, la cual asume que la densidad es invariante en el volumen de control, esto realmente implica considerar fluido incompresible; es decir, se desprecian las variaciones de la densidad excepto en el caso que afecta a la fuerza gravitatoria, que da origen a la fuerza de flotación, posteriormente considerada en la ecuación de conservación de cantidad de movimiento.

Se considera una modelación en régimen permanente, turbulento, con flujo Newtoniano e incompresible, con propiedades físicas constantes. La ecuación en variables promediadas y en régimen permanente para la conservación de la masa (continuidad) (1), cantidad de movimiento (2) y energía (3) pueden expresarse de la siguiente forma (Tennekes y Lumley , [1972]):

$$
\begin{aligned}
& \frac{\partial}{\partial x_{j}} u_{j}=0 \\
& \rho \frac{\partial}{\partial x_{j}} u_{i} u_{j}=-\frac{\partial p}{\partial x_{i}}+\frac{\partial}{\partial x_{j}} \mu\left(\frac{\partial u_{i}}{\partial x_{j}}+\frac{\partial u_{j}}{\partial x_{i}}\right)-\frac{\partial}{\partial x_{j}} \rho\left(\overline{u_{i}^{\prime} u^{\prime}}\right)+F_{i}
\end{aligned}
$$

$$
\rho C_{p} \frac{\partial}{\partial x_{j}} T u_{j}=\frac{\partial}{\partial x_{j}}\left(-k \frac{\partial T}{\partial x_{j}}\right)+\frac{\partial}{\partial x_{j}} \rho C_{p}\left(u_{j}^{\prime} T^{\prime}\right)
$$

Donde $\mathrm{u}_{\mathrm{i}^{\prime}} \mathrm{T}$ y p son valores medios, mientras que $u^{\prime}{ }_{j}$ y $T^{\prime}$ son componentes fluctuantes de la velocidad y temperaturas instantáneas. Las velocidades medias y fluctuantes se definen, tal como muestra la velocidad en las ecuaciones (4), con $v_{i}$ un valor instantáneo.

$u_{i}=\frac{1}{\Delta t} \int_{t}^{t+\Delta t} v_{i} d t \quad u_{i}^{\prime}=v_{i}-u_{i}$

Las expresiones contenidas entre paréntesis $\left(\overline{\mathrm{u}_{i}^{\prime} \mathrm{u}_{j}^{\prime}}\right)$ y $\rho \mathrm{C}_{\mathrm{p}}\left(\overrightarrow{\mathrm{u}^{\prime} \mathrm{T}^{\prime}}\right)$ representan la promediación de dos cantidades fluctuantes. Estas expresiones tienen el sentido físico de representar las Tensiones de Reynolds y el flujo adicional 
debido a fenómenos turbulentos. $F_{i}$ en este caso es la componente de fuerza de empuje vertical debidas a las diferencias de densidad entre los flujos de aire a diferentes temperaturas.

Asimismo, la tensión de Reynolds se relaciona con los valores medios de las variables mediante las hipótesis de Boussinesq:

$\rho\left(\overline{u_{i}^{\prime} u^{\prime}}\right)=\frac{2}{3} \rho k_{e} \delta_{i, j}-\mu_{t}\left(\frac{\partial u_{i}}{\partial x_{j}}+\frac{\partial u_{j}}{\partial x_{i}}\right)$

Siendo $k_{e}$ la energía cinética turbulenta definida como:

$$
\mathrm{k}_{\mathrm{e}}=\frac{1}{2}\left(\overline{\mathrm{u}_{\mathrm{i}}^{\prime} \mathrm{u}_{\mathrm{j}}^{\prime}}\right)
$$

Y el término de flujo de calor debido a la turbulencia en la ecuación (3) análogamente se representa con la viscosidad turbulenta en la ecuación (7)

$$
\rho C_{p}\left(\overline{u^{\prime}{ }_{j} T^{\prime}}\right)=k_{t}\left(\frac{\partial T_{i}}{\partial x_{i}}\right)
$$

Donde $\mu_{t}$ y $k_{t}$ son propiedades del flujo, que se encuentran conectadas mediante el número de Prandtl. La bibliografía consultada propone un numero de Prandtl adecuado en este caso de 0.9 ,(White, [1991]).

En este caso, todas las condiciones y propiedades han sido definidas en el software comercial STAR-CCM+ y resueltas utilizando su código acoplado. Los resultados se presentan mediante las técnicas de postproceso del citado código. Los resultados analizados incluyen los siguientes modelos de transferencia: difusión y convección natural en el volumen modelado

En este caso la geometría modelada es tridimensional y considerado en régimen llamado permanente: no se consideran variaciones temporales en tanto que se representan situaciones "fijas"

Con respecto a la resolución de las ecuaciones se utiliza el llamado "Modelo de resolución segregado".

El modelo segregado resuelve las ecuaciones del flujo (una para cada componente de la velocidad y una para la presión) de forma desacoplada. La unión entre las ecuaciones de continuidad y conservación de cantidad de movimiento se realiza en este caso con una aproximación del tipo predictorcorrector en los volúmenes finitos que se han definido para la resolución numérica de las ecuaciones (STAR CCM+ Manual). Este modelo tiene su origen y completa aplicación en casos de flujo incompresible o así considerado, como el que nos ocupa.

Frente al método "segregado" se encuentra el método de resolución "acoplada", donde las ecuaciones de conservación se resuelven simultáneamente, sin tener que recurrir a aproximaciones. Este método requiere, por tanto, la solución de grandes sistemas de ecuaciones acopladas de forma simultánea.

El llamado "modelo con gravedad" permite la inclusión de fenómenos de flotación en los movimientos del fluido vertical, necesario en este caso. La aproximación de Boussinesq es la que se usa en este caso.

La turbulencia debe ser considerada mediante una aproximación de cierre adecuada.
En la presente modelación, la solución de cierre que mejor se adapta a las condiciones de modelación es el de k-ع. Este es un modelo de dos ecuaciones en las que para conseguir las soluciones de las ecuaciones se hace necesario definir dos nuevos parámetros, responsables del cierre de las ecuaciones: la energía cinética turbulenta y la disipación de energía cinética turbulenta.

La bibliografía consultada y el grado de convergencia adquirido para los residuos de la turbulencia en esta modelación a juicio de los autores justifican el uso de esta solución de cierre frente a otras que se han presentado. Asimismo, la física del problema permite el uso de ecuaciones con premediación de Reynolds y soluciones de cierre en este ámbito.

Varios son los modelos que aquí se pueden tener en cuenta a la hora de abordar el problema del cierre (Gualtieri et al., [2009]; Gualtieri et al, [2010]; Wilkox, [2002]; Hanjalić, [2004]):

- Modelos de una ecuación. En este caso, se resuelve una sola ecuación diferencial para $k$. Un ejemplo de ellos es el modelo de Spalart-Allmaras

- Modelos de dos ecuaciones. De forma adicional a la anteriormente descrita para $k$, debe resolverse una ecuación más para proporcionar la tasa de disipación de energía cinética turbulenta $\varepsilon$, y tasa de disipación específica $\omega=\varepsilon / \mathrm{k}$ que es la variable que determina la escala de la turbulencia, Wilcox, [2002]. Los así llamados modelos $\mathrm{k}-\varepsilon$, junto con $\mathrm{k}-\omega$ y sus variaciones, son adecuados para la representación de este caso. Particularmente una de las ventajas del modelo $k-\omega$ frente a $k-\varepsilon$ es su mejor comportamiento en la modelación de los procesos en capa límite, incluso bajo gradientes de presión adversos, sin embargo, el modelo k- $\varepsilon$ presenta una capacidad de convergencia aceptable especialmente con variaciones de presión no muy marcadas.

- Modelos de los esfuerzos o Tensiones de Reynolds (RSM por sus siglas en inglés Reynolds Stress Model). Estos son modelos denominados DSM (Differential Second-Moment turbulence models), que tienen un grado mayor de exactitud inherente a su solución de cierre. En este caso no se abordan las ecuaciones promediadas para la turbulencia, como los anteriores de una o dos ecuaciones con nuevas incógnitas, sino que el tratamiento es más complejo puesto que se pretende establecer una ecuación diferencial para cada una de las Tensiones de Reynolds modelando los términos de producción, transporte, difusión y rotación. Así pues, estos modelos a priori se presentan más adecuados para procesos en los que se den fenómenos de rotación, fuerte curvatura de las líneas de corriente o flujos secundarios y anisótropos. La desventaja de este modelo frente a los más tradicionales es el costo computacional que representan. En modelaciones tridimensionales deberán ser resueltas siete ecuaciones mientras que en modelos de dos dimensiones, se resuelven cinco ecuaciones simultáneamente (Sarkar y Balakrishnan, [1990], Speziale et al. (1997), STAR-CCM+ [2010]). En este caso, dada la clara direccionalidad del flujo, se desconsidera el uso de esta solución de cierre. 
Así pues, el modelo k-e considera una distribución de la viscosidad turbulenta obtenida como

$$
\mu_{\mathrm{t}}=\rho \mathrm{C}_{\mu}\left(\frac{\mathrm{k}_{\mathrm{e}}}{\varepsilon}\right)
$$

Donde $C_{\mu}=0,09$ (Launder, [1972]).

La energía cinética turbulenta $k_{e}$ y su disipación $\varepsilon$ se conocen resolviendo las ecuaciones clásicas para las mismas (Launder, [1972]).

$$
\begin{aligned}
& \frac{\partial}{\partial \mathrm{x}_{\mathrm{j}}} \rho \mathrm{u}_{\mathrm{j}} \mathrm{k}_{\mathrm{e}}=\frac{\partial}{\partial \mathrm{x}_{\mathrm{j}}} \frac{\mu_{\mathrm{t}}}{\sigma_{\mathrm{k}}} \frac{\partial \mathrm{k}_{\mathrm{e}}}{\partial \mathrm{x}_{\mathrm{j}}}+\left(-\rho \overline{\left(\mathrm{u}_{\mathrm{i}}^{\prime} \mathrm{u}^{\prime}\right.}{ }_{\mathrm{j}} \frac{\partial \mathrm{u}_{\mathrm{j}}}{\partial \mathrm{x}_{\mathrm{i}}}+\beta \mathrm{g}_{\mathrm{i}} \frac{\mu_{\mathrm{t}}}{\operatorname{Pr}_{\mathrm{t}}} \frac{\partial \mathrm{T}}{\partial \mathrm{x}_{\mathrm{i}}}-\rho \varepsilon\right)(9) \\
& \frac{\partial}{\partial \mathrm{x}_{\mathrm{j}}} \rho \mathrm{u}_{\mathrm{j}} \varepsilon=\frac{\partial}{\partial \mathrm{x}_{\mathrm{j}}} \frac{\mu_{\mathrm{t}}}{\sigma_{\varepsilon}} \frac{\partial \varepsilon}{\partial \mathrm{x}_{\mathrm{j}}}-\mathrm{C}_{\varepsilon 1} \rho \overline{\left(\mathrm{u}_{\mathrm{i}}^{\prime} \mathrm{u}_{\mathrm{j}}^{\prime}\right.} \frac{\partial \mathrm{u}_{\mathrm{j}}}{\partial \mathrm{x}_{\mathrm{i}}} \frac{\varepsilon}{\mathrm{k}_{\mathrm{e}}}-\mathrm{C}_{\varepsilon 2} \rho \frac{\varepsilon^{2}}{\mathrm{k}_{\mathrm{e}}}
\end{aligned}
$$

Las constantes que se utilizan adoptan el valor propuesto en la bibliografía:

$$
C \varepsilon 1=1,44 ; C \varepsilon 2=1,92 ; \sigma_{k}=1,0 ; \sigma_{\varepsilon}=1,3
$$

\subsection{Metodología aplicada para la realización del modelo matemático}

El presente estudio se realiza con objeto de cuantificar el efecto que la fachada ventilada tiene sobre el ahorro de energía en la estancia en la cual se instala en referencia a las necesidades de energía para refrigeración.

Así pues, se plantea un balance de calor en el entorno de la fachada. El objetivo es conocer la temperatura que tendría la pared directamente en contacto con la estancia cuando en su cara exterior en contacto con el ambiente existe la fachada ventilada en comparación con la no existencia de la misma. De esta forma, en la Figura 4 se representa la geometría considerada para la pared donde se aplica el modelo.

Se ha obviado la contribución del material aislante en la cara exterior del cerramiento, puesto que el objeto de la investigación es determinar si es posible reducir, o incluso anular, la necesidad de utilizar dicho aislamiento térmico gracias a la contribución a la eficiencia energética proporcionada por la fachada ventilada.

\subsubsection{SIMPLIFICACIONES}

El objetivo que se persigue en la presente contribución es mostrar la capacidad del código CFD para modelar los aspectos de la mecánica del flujo en el seno de la fachada ventilada y proporcionar una herramienta para comprender el papel que juega el panel cerámico en la posible mejora energética y ahorro de refrigeración en la estancia interior. Por ello, se han realizado algunas simplificaciones a la hora de plantear una comparación entre la fachada ventilada y la que no lo está, que se indican a continuación:

a. En tanto que el interés se centra en la lámina entre los paneles interior y exterior, se ha considerado la misma temperatura exterior en el ambiente y en la superficie del edificio en contacto con el mismo. Esto de hecho, depende del material y los aspectos de convección y radiación de cada caso, de los colores de los materiales y su posición geográfica frente al sol, junto con el régimen de vientos y la posibilidad de estar mojados por lluvias o diferentes grados de humedad. Estos hechos se han desconsiderado tomando las mismas condiciones, a modo de simplificación.

b. En el cálculo de la transferencia de calor a través de la hoja interna no se tendrá en cuenta la capacidad de la misma para acumular energía térmica ("inercia térmica"). Se conoce que esta inercia es importante en diferentes cerramientos exteriores, en la presente contribución se ha supuesto que el muro interior va a tener el mismo comportamiento en todos los casos, sin tener en cuenta otros aspectos térmicos constructivos que permitan acumulaciones temporales de calor. La inercia térmica, considerada como la propiedad que indica la cantidad de calor que puede conservar un cuerpo y la velocidad con que la cede o absorbe del entorno dependería de la masa, del calor específico de sus materiales y del coeficiente de conductividad térmica de éstos; parámetros que no se varían en el muro interior en este modelo simplificado con y sin paneles cerámicos de fachada exterior.

c. La modelación se ha considerado en régimen permanente, con unas condiciones de contorno de temperaturas favorables al efecto de enfriamiento adicional que supone la fachada ventilada. No se han considerado estaciones del año ni variabilidad diurna y nocturna, que pondrán la eficiencia de la fachada en otros valores. La consideración de régimen permanente tiene como consecuencia que la inercia térmica no se considere. Esta simplificación es importante en tanto que en la realidad se conoce que afectará fuertemente al ahorro que supone la fachada ventilada.

d. En este caso, se ha considerado que el panel de cerámica exterior se encuentra sometido a la acción solar de manera que esta es una fuente de calor suficientemente grande y constante en la consideración de régimen permanente que se ha realizado como para mantener la temperatura del panel cerámico, como sólido, constante en toda la altura.

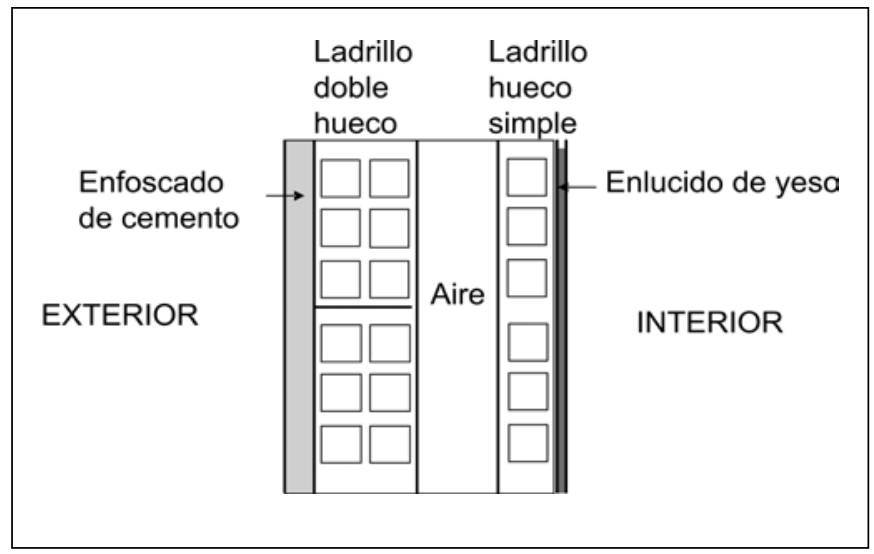

Figura 4. Muro estándar considerado para la modelación 


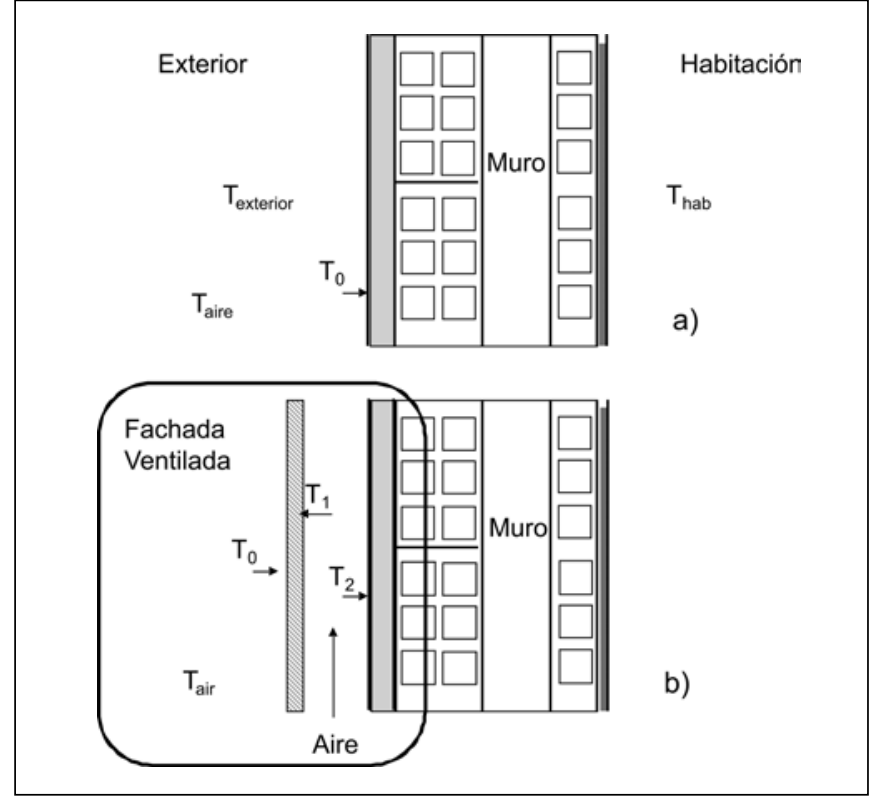

Figura 5. Nomenclatura de temperaturas para la simulación

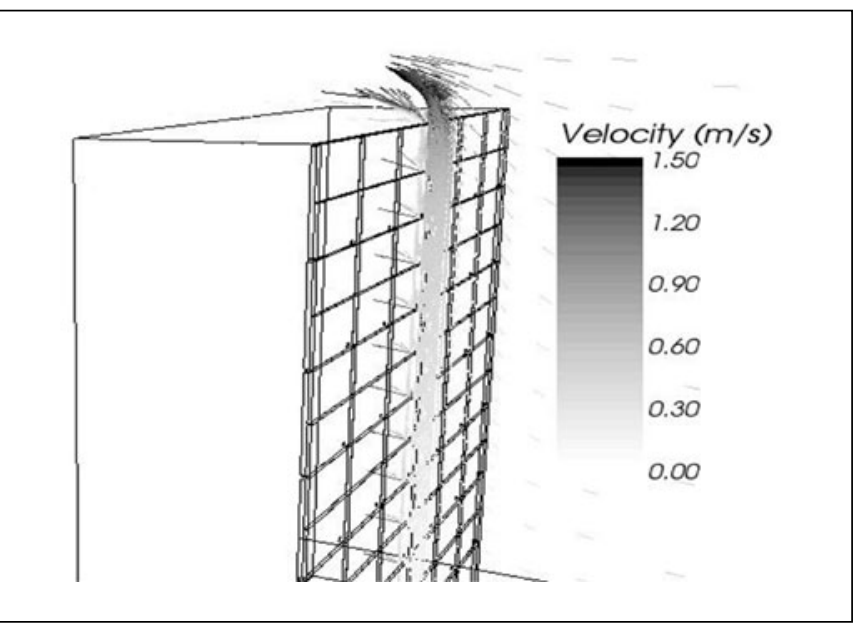

Figura 6. Perfil de velocidades en la línea intermedia considerado en la modelación $(\mathrm{m} / \mathrm{s})$

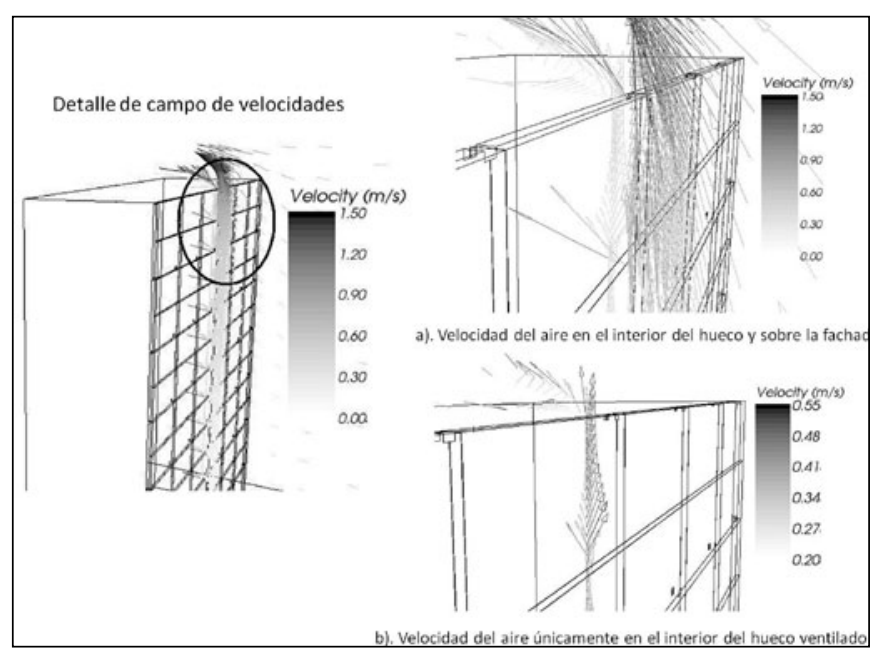

Figura 7. Detalle del campo de velocidades.
La Figura 5 representa las temperaturas consideradas en el muro representado, para comparar las situaciones en las cuales no se instala la fachada ventilada (a) y en el caso en que se modela la presencia de los paneles exteriores (b). En este caso, para el modelo numérico, la temperatura del aire $T_{\text {air }}$ en primer lugar ha sido fijada a $298 \mathrm{~K}\left(25^{\circ} \mathrm{C}\right)$. La temperatura interior de los paneles de la fachada ventilada ha sido medida en laboratorio con condiciones semejantes y fijada para la modelación a $\left(\mathrm{T}_{1}\right) 304 \mathrm{~K}\left(31^{\circ} \mathrm{C}\right)$. Con estas consideraciones, modelamos la temperatura de la pared $\mathrm{T}_{2}$, directamente en contacto con el muro exterior de la estancia que desearíamos acondicionar. Dentro de esta habitación se desearía mantener 296K $\left(23^{\circ} \mathrm{C}\right)$, así llamada $\mathrm{T}_{\text {inside }}$.

\subsection{Resultados de la modelación: Campo de velocidades y temperaturas}

El modelo computacional de la mecánica de fluidos CFD calculará la velocidad del aire en el hueco que disponen los paneles de la fachada, dependiendo de la velocidad del viento en la envolvente del edificio y la capacidad convectiva del aire debida a la diferencia de temperaturas. Se ha considerado una velocidad del aire exterior perpendicular a la fachada, las velocidades en el hueco se calculan por el código computacional propuesto, como se ilustra en Figura 6, cuando $\left(\mathrm{T}_{1}\right)$ es $304 \mathrm{~K}\left(31^{\circ} \mathrm{C}\right)$. En esta figura se presenta el perfil de velocidades en la sección centrada en la tercera columna de paneles desde la izquierda. En ella puede verse cómo el campo de velocidades vectorial tiende a oscurecerse (valores mayores de velocidad) hacia la parte superior del edificio.

$<$ El aire puede entrar en la fachada por las holguras que existen entre las piezas, y entre ellas y la base del edificio. Las ranuras tienen una anchura de $2 \mathrm{~mm}$. Los laterales de la fachada se encuentran abiertos, sin embargo la condición de contorno definida con una velocidad del viento perpendicular a los paneles de fachada, la velocidad en el contorno tangente al mismo, no genera entrada a través de los citados laterales.

Para mostrar más detalle sobre el campo de velocidades en esta zona central de la fachada, donde los contornos laterales no influyen, se incluye la Figura 7. La citada figura muestra dos detalles del campo de velocidades en la zona superior del

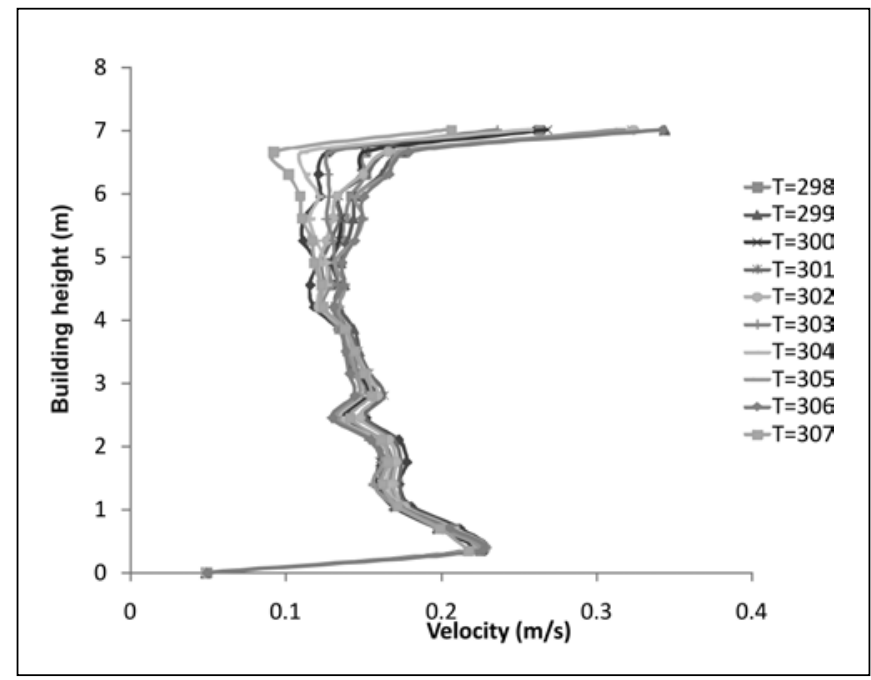

Figura 8. Valores escalares de la velocidad para diferentes $\mathrm{T}_{1}$. 
edificio. La Figura 7a) detalla la velocidad en toda esta zona de computación, donde el flujo entra por las aberturas; así como el campo de velocidades en el hueco entre los paneles de la fachada ventilada. La Figura $7 b$ ) muestra únicamente el campo de velocidades en este intersticio que se describe, mayormente vertical hacia arriba.

Estas velocidades dependen de la temperatura $\mathrm{T}_{1}$, conforme esta cambia, el efecto convectivo hace que la velocidad del aire que asciende en el hueco de la fachada ventilada, también lo haga. Para ilustrar este hecho, se presenta la Figura 8 que indica el módulo de la velocidad a la que asciende el aire por la línea central de la fachada ventilada en la sección presentada en la Figura 8.

Con estas consideraciones de temperatura y las velocidades modeladas en dirección perpendicular al muro, el modelo numérico determina la temperatura $T_{2^{\prime}}$ directamente en contacto con el muro de la habitación a modelar, y cuyo valor depende de la eficiencia de la capacidad de refrigeración de la fachada. Esta temperatura en el conjunto de la pared frontal se muestra en la Figura 9.

Considerando que la temperatura de la cara interior del panel es constante: $T_{1}=304 \mathrm{~K}$; esto significa que la temperatura ha decrecido en el hueco de la fachada, como consecuencia de la ventilación. Esta disminución media en el hueco de la fachada, en este caso particular es de $4,482 \mathrm{~K}$, a pesar de que esta temperatura va variando con la altura conforme el aire asciende, como se muestra en la Figura 10.

La disminución de temperatura en la cara externa del muro en contacto con el hueco de aire de la fachada,

$\mathrm{T}_{2}$ supondrá por tanto, un ahorro energético en la necesidad de refrigeración de la estancia en contacto con la misma.

Los estudios desarrollados por Chiampi en [2003] muestran que uno de los factores que más afectan a la eficiencia de la fachada es la temperatura del aire exterior. En condiciones de verano, los ahorros energéticos aumentarán considerablemente conforme lo haga la radiación solar y por tanto se forzará el movimiento convectivo del aire y la capacidad de refrigeración del aire que circula en el hueco de la fachada. Este aspecto se considera importante y ha sido asimismo, en este caso particular, modelado para la fachada cerámica ventilada representada utilizando el análisis aerodinámico con CFD presentado. La Figura 11 muestra la variación de temperatura en el hueco de la fachada cuando variase la temperatura $T_{1}$ como consecuencia del calentamiento del panel cerámico externo de la fachada y simulando consecuentemente $T_{2}$ en cada situación. Como puede observarse en la Figura 10, conforme la temperatura del panel externo aumenta, la caída de temperatura en el hueco de aire es mayor $\left(\mathrm{T}_{1}-\mathrm{T}_{2}\right)$ y el efecto de refrigeración pasiva generado por la fachada cerámica ventilada es más efectivo. Con la temperatura en cada modelación obtenida en una línea vertical como se indica en la Figura 11, (pegada al panel cerámico y considerando que $\mathrm{T}_{1}$ es fija para cada modelación), se ha calculado la variación de temperatura en el seno del hueco ventilado.

Con los datos ambientales de esta modelación, se observa que el efecto de refrigeración del aire en el hueco de la fachada cerámica tiene un comportamiento lineal. Tal como se ha presentado en la Figura 11, la Figura 12 muestra el descenso medio de temperatura en el hueco de la fachada cerámica cuando cambia la temperatura del panel exterior en las mismas condiciones que el modelo anterior.

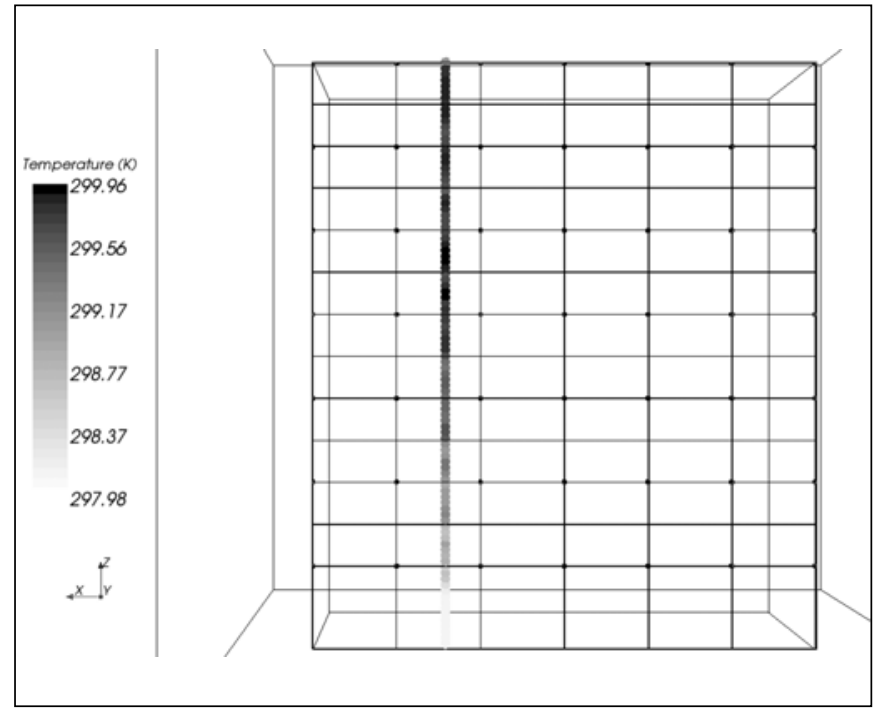

Figura 9. Distribución de temperaturas $\mathrm{T}_{2}$ en el muro protegido por la fachada

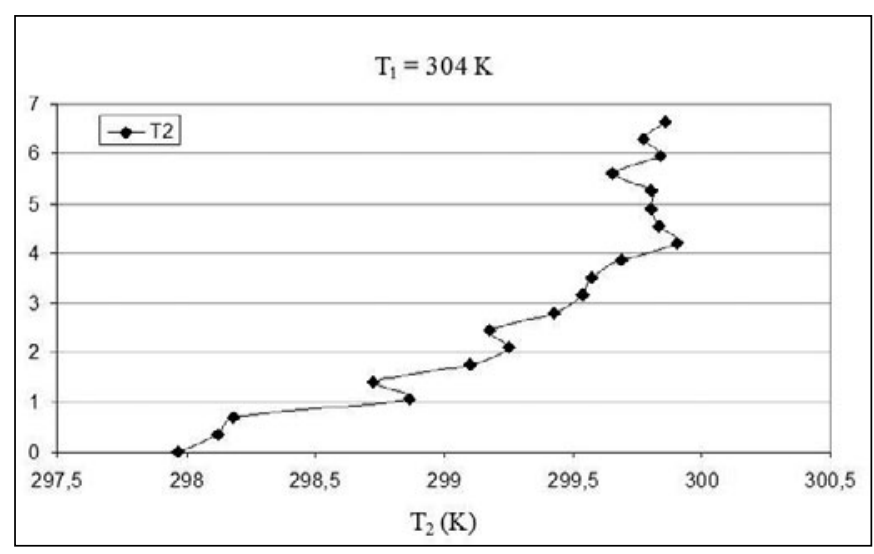

Figura 10. Evolución vertical de $\mathrm{T}_{2}$ en el edificio modelado

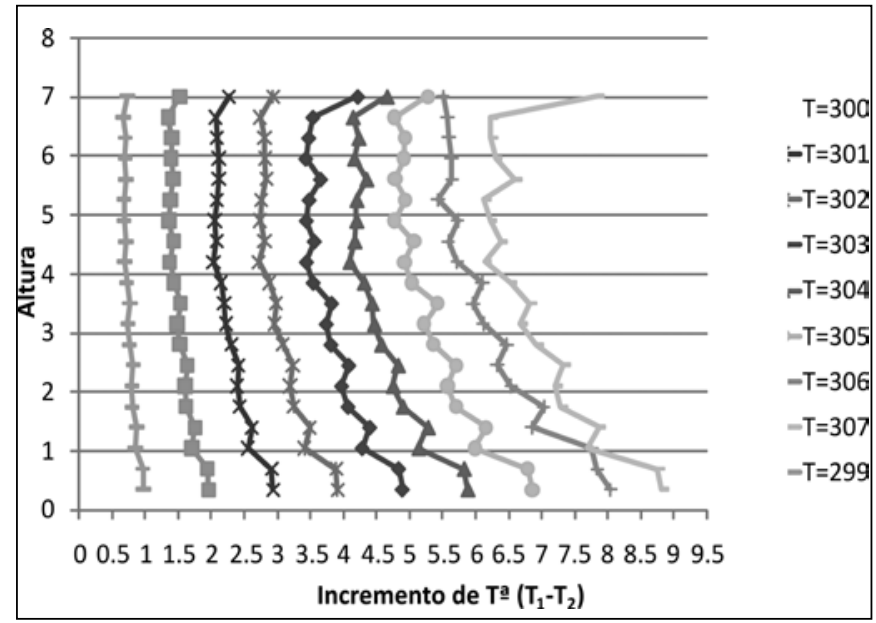

Figura 11. Variación de temperatura en el hueco del aire modelada por el código CFD cuando cambia $\mathrm{T}_{1}$ 
De la misma forma que la variación de la radiación exterior al variar $T_{1}$ afecta a la temperatura interior, también el efecto convectivo del calentamiento afecta consecuentemente a la velocidad en el interior del hueco de aire, como se ha mostrado en las figuras que representan los campos vectoriales anteriormente citadas.

\subsection{Análisis energético}

Desde un punto de vista de la determinación de la eficiencia, el objetivo principal de la fachada cerámica ventilada sería, tal como se plantea en la presente contribución, un ahorro de la energía de refrigeración que habría que transmitir a la estancia en la que se instala la fachada al instalar los paneles cerámicos externos. Este tema ha sido estudiado recientemente y reflejado en la bibliografía: Gang (1998), Manz [2003], Balocco, [2003].

Los análisis energéticos así propuestos, implican un conocimiento de la transmisión de calor a través del muro que cierra la estancia. Por ello es necesario conocer un valor para la modelación del coeficiente de transmisión de calor U. Se requiere información sobre los valores de coeficientes de transitividad de todas y cada una de las capas que componen la fachada y una modelación considerando la transmisión de calor en la pared sólida más profunda que la que aquí se presenta como primera aproximación. Para proponer una estimación primera y demostrar la ayuda que puede prestar al diseñador la modelación mediante la técnica CFD presentada, se propone un análisis comparativo

Con el modelo en condiciones de verano que se ha realizado, y entendiendo que se desea mantener en el interior de la estancia una temperatura de $296 \mathrm{~K}\left(23{ }^{\circ} \mathrm{C}\right)$, podemos conocer el calor transmitido a través del muro que se encuentra en contacto con la fachada. La bibliografía consultada propone diferentes valores para este coeficiente Yilmaz, Z. [2007], comprendidos entre 0.55 y 1.42. La Tabla 1 ha sido construida considerando unos valores para los coeficientes de conductividad térmica de los materiales $\mathrm{k}$ indicados y unos coeficientes de convección superficial interior y exterior $\mathrm{h}$ de $10 \mathrm{~W} / \mathrm{m}^{2} \cdot \mathrm{K}$. el valor de la transmitancia de calor $\mathrm{U}$ completo para el cerramiento se ha calculado siguiendo la expresión (11). Los valores para los coeficientes representativos de los diferentes elementos se han tomado de valores pro defecto utilizados en las modelaciones (CTE, [2010]).

\section{Comparación Incrementos}

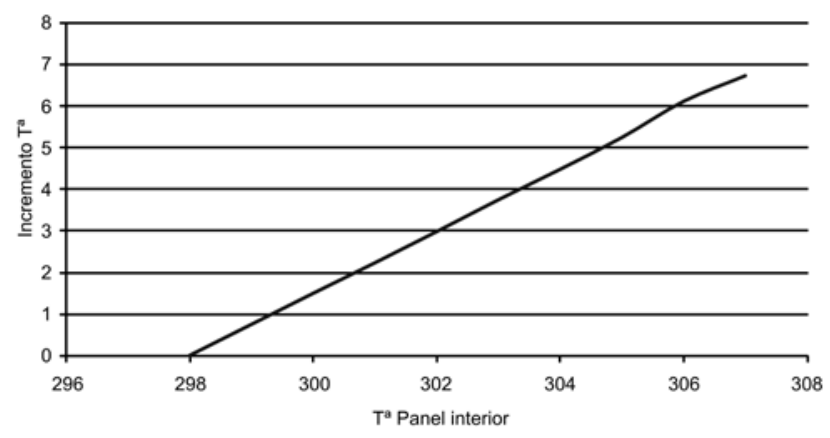

Figura 12. Variación de temperatura media $\mathrm{T}_{2}$ modelada por el código CFD cuando cambia $\mathrm{T}_{1}$

$$
\mathrm{U}=\frac{1}{\left(\frac{1}{\mathrm{~h}_{\mathrm{i}}}+\sum\left(\frac{\mathrm{e}_{\mathrm{i}}}{\mathrm{k}_{\mathrm{i}+}}\right)+\frac{1}{\mathrm{~h}_{\mathrm{e}}}\right)}
$$

TABla 1. CONSIDERACIONES PARA El CÁlCUlO DEL COEFICIENTE DE TRANSMISIÓN DE CALOR DEL CERRAMIENTO.

Coeficiente Global

Transmisión de Calor

\begin{tabular}{|ccc|}
\hline h_interior & 10 & $\mathrm{~W} / \mathrm{m}^{2} \cdot \mathrm{K}$ \\
\hline (e/k) & 0,71 & $\mathrm{~m}^{2} \cdot \mathrm{K} / \mathrm{W}$ \\
\hline h_exterior & 10 & $\mathrm{~W} / \mathrm{m}^{2} \cdot \mathrm{K}$ \\
\hline & & \\
\hline $\mathbf{U}$ & $\mathbf{1 , 1 0}$ & $\mathbf{W} / \mathbf{m}^{\mathbf{2}} \cdot \mathbf{K}$ \\
\hline
\end{tabular}

Materiales fachada "TIPO" edificio

\begin{tabular}{|l|c|c|c|}
\cline { 2 - 4 } \multicolumn{1}{c|}{} & $\begin{array}{c}\mathrm{e} \\
(\mathrm{m})\end{array}$ & $\begin{array}{c}\mathrm{k} \\
(\mathrm{W} / \mathrm{m} \cdot \mathrm{K})\end{array}$ & $\begin{array}{c}\mathrm{e} / \mathrm{k} \\
\left(\mathrm{m}^{2} \cdot \mathrm{K} / \mathrm{W}\right)\end{array}$ \\
\hline Enfoscado de cemento & 0,02 & 1,2 & 0,017 \\
\hline Ladrillo doble hueco & 0,12 & 0,42 & 0,29 \\
\hline Cámara de aire & 0,04 & 0,19 & 0,21 \\
\hline Ladrillo hueco simple & 0,05 & 0,42 & 0,12 \\
\hline Enlucido de yeso & 0,02 & 0,26 & 0,08 \\
\hline
\end{tabular}

Obteniendo así un valor para el coeficiente global de transmisión de calor de $1,1 \cdot \frac{\mathrm{W}}{\mathrm{m}^{2} \mathrm{~K}}$

La temperatura de la pared donde no se instala la fachada, en las mediciones realizadas para esta situación, se considera de $305 \mathrm{~K}\left(32^{\circ} \mathrm{C}\right)$; el calor transferido (q) a través de la pared, será:

En el caso de la fachada sin ventilar

$$
\mathrm{q}=\mathrm{U}_{\text {wall }} \cdot(\Delta \mathrm{T})=1,1 \cdot\left(\mathrm{T}_{0}-\mathrm{T}_{\text {hab }}\right) \frac{\mathrm{W}}{\mathrm{m}^{2}}
$$

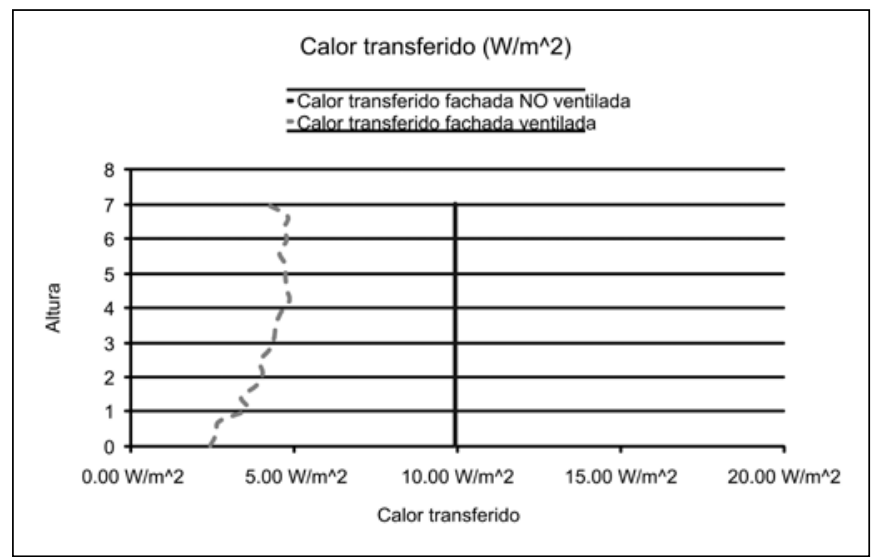

Figura 13. Calor transferido a través de la fachada para mantener la temperatura interior en $296 \mathrm{~K}$. 
En el caso de la fachada ventilada, con estas condiciones:

$$
\mathrm{q}=\mathrm{U}_{\text {wall }} \cdot(\Delta \mathrm{T})=1,1 \cdot\left(\mathrm{T}_{2}-\mathrm{T}_{\text {hab }}\right) \frac{\mathrm{W}}{\mathrm{m}^{2}}
$$

Un análisis simplificado de la simulación anterior, muestra la diferencia de calor transferido desde el exterior hasta el interior de la estancia con la existencia de la fachada y sin la existencia de la misma en las condiciones de la simulación.

La Figura 13 representa el efecto en el gasto energético que tendremos para mantener la temperatura en la estancia en 296K. Analizando esta figura, se observa que si no se dispone de la fachada ventilada, para mantener las condiciones de temperatura en la estancia interior, se transfieren $9,9 \mathrm{~W} / \mathrm{m}^{2}$. Sin embargo, si se dispone de la fachada, (en las condiciones puntuales de la modelación), el calor transferido es menor: $4,09 \mathrm{~W} / \mathrm{m}^{2}$ en el conjunto de la altura del edificio: el efecto del enfriamiento del muro es que la energía en este momento para mantener la temperatura interior es un $58,7 \%$ menos si se dispone de la fachada ventilada que si no se dispone de la misma.

Este ahorro energético, obviamente depende de la situación térmica externa. Tal como aquí se ha presentado, cuando la temperatura del aire exterior baje, el efecto de ahorro energético de la fachada ventilada disminuye, llegando a ser innecesario cuando la temperatura deseada en habitáculo es de forma natural, la que tiene el aire exterior. Asimismo, en este análisis instantáneo no ha considerado la inercia térmica de las paredes y los materiales, al tratarse de un estudio puntual en régimen permanente, pero este efecto será importante en el análisis del ahorro medio diario y estacional.

Los resultados que aquí se presentan se encuentran en la línea de las investigaciones más recientes sobre la fachada cerámica ventilada. El artículo presentado por Ciampi et al, en [2003] se presenta una comparativa entre el efecto de la fachada ventilada con el aire en movimiento y la capa adicional de material aislante tradicional con que habría que cargar la fachada no ventilada para conseguir el mismo efecto (Ciampi, [2003]) de una forma analítica. De forma complementaria a este análisis, se propone el presente estudio basado en la mecánica de fluidos computacional para estimar el efecto de ahorro energético de la fachada cerámica ventilada

\section{CONCLUSIONES}

- La cuantificación del efecto que supone la instalación de una fachada cerámica ventilada en una edificación es compleja. No se dispone en la bibliografía de parámetros directos para la valoración de dicha eficiencia. En la presente contribución se propone una estrategia de cuantificación de este efecto bajo ciertas condiciones utilizando para ello la modelación de la fluidodinámica computacional: CFD.

- Se ha descrito la aplicación de la resolución computacional de las ecuaciones de la mecánica de fluidos para el aire que ocupa el hueco que resulta en la fachada cerámica ventilada. En un caso de estudio con temperaturas tomadas de ensayos en laboratorio, se ha representado una situación plausible en climas mediterráneos en condiciones de verano. En este caso se ha calculado la temperatura del aire y el enfriamiento que para el muro de cerramiento de una estancia puede suponer la instalación de los paneles de la fachada cerámica ventilada y se ha podido conocer la temperatura en todo el volumen de aire; y por tanto en la cara exterior del muro. Utilizando este perfil de temperaturas, en estas condiciones para la modelación, se ha planteado una cuantificación de la energía de refrigeración que se ahorra al comparar la utilización de la fachada con el caso en que no se implemente y se puede cuantificar un ahorro posible $58,7 \%$ en la energía para mantener la temperatura interior de la estancia. Esta gran cantidad se ha conseguido con la suposición de los campos de temperaturas externas tal como se ha presentado y en régimen permanente, máximo cuantificable en estas condiciones.

- Más allá de la proposición de la presente metodología para conocer la temperatura del aire interior de la fachada, la técnica de la fluidodinámica computacional se presenta como una herramienta potente para la representación del efecto en el comportamiento en la envolvente del edificio que tiene la instalación de la fachada cerámica ventilada. Sin la ayuda de esta modelación computacional es imposible calcular de forma continua las temperaturas y velocidades de fluidos como el aire, que juega un papel fundamental en este caso.

- Se hace, pues, patente que se requiere una modelación matemática más compleja en la que se integren los fenómenos de transmisión de calor en los sólidos del muro y los paneles. Hay muchos parámetros de decisión que influyen en la eficiencia de esta instalación: la separación entre los paneles, la temperatura y velocidad del aire entrante, la radiación solar y temperaturas de los paneles sólidos, los coeficientes de transmisión de calor de los elementos sólidos, etc. Sin embargo, esta modelación computacional permitirá realizar un análisis de sensibilidad sencillo variando estos parámetros que, con las actuales capacidades de computación, serán rápidos y permitirán determinar las soluciones óptimas de funcionamiento, especialmente si se integra el fenómeno de la radiación en los elementos sólidos del muro y puede hacerse un análisis no permanente que integre la inercia térmica.

- En esta línea, la primera aproximación presentada en esta contribución postula a la fluidodinámica computacional como una técnica de evaluación del comportamiento de la fachada muy adecuada y completa. En el futuro, tras las pertinentes calibraciones en condiciones controladas, permitirá conocer las mejores condiciones de instalación y selección de fachadas cerámicas ventiladas para optimizar su comportamiento desde el punto de vista energético incluso antes de su instalación. Asimismo, es claro que los resultados de la modelación numérica pertenecen al campo de las simulaciones, que tienen que ser validadas con resultados reales, que deberán ser desarrollados en el futuro, de forma complementaria a la presente investigación. 


\section{LISTA DE SÍMBOLOS}

$\mathrm{C}_{\varepsilon 1}$; constante del modelo CFD

$\mathrm{C}_{\varepsilon 2}$ constante del modelo CFD

$\mathrm{C}_{\mathrm{p}}$ Calor específico a presión constante para el aire

$\mathrm{F}$ fuerza de empuje vertical

g aceleración de la gravedad

h Coeficiente de convección superficial

k Coeficiente de Conductividad térmica

$\mathrm{k}_{\mathrm{e}}$ energía cinética turbulenta

kt conductividad turbulenta

$\mathrm{p}$ presión media

$\mathrm{P}_{\mathrm{r}}$ Número de Prandtl

q Calor transferido

T temperatura

U Transmitancia de calor

$u_{j}$ componente de la velocidad

$\varepsilon$ Disipación de energía cinética turbulenta

$\mu$ viscosidad

$v_{i}$ valor instantáneo de la velocidad

$\rho$ densidad del aire

\section{AGRADECIMIENTOS.}

La realización del trabajo de investigación que aquí se describe se enmarca dentro de las acciones de la Ayuda: "Aplicación de técnicas de la mecánica de Fluidos Computacional al Movimiento de Flujos Ambientales" dotada por la Universidad Politécnica de Valencia.

\section{REFERENCIAS}

1. C. Balocco, A simple model to study ventilated façades energy performance. Energy and Buildings, Vol 34, N 4. Pp 469-475. 2002

2. C. Balocco, A non dimensional analysis of a ventilated double façade energy performance. Energy and Buildings, Vol 36, N 1. Pp 35-40. 2003

3. M. Ciampi; F. Leccese; G. Tuoni, Ventilated façades energy performance in summer cooling of buildings. Solar Energy N. 75. Pp 491-502. 2003.

4. CTE. Codigo Técnico de la Edificación. Documento Básico. Ahorro de energía. 2010. Consultado en Internet en la dirección:
5. http://www.codigotecnico.org/cte/export/sites/default/web/galerias/ archivos/DB_HE_abril_2009.pdf

6. H. El Sadi; Haghighat; and Ali Fallahi. (2010). CFD Analysis of Turbulen Natural Ventilation in Double-Skin Façade: Thermal Mass and Energy Efficiency. Journal of Energy Engineering 136, 68-75.

7. G. Gang, A parametric study of Tromble walls for passive cooling of buildings, Energy and Buildings, Vol 27, N 1. Pp 37-43. 1998.

8. C. Gualtieri, P.A. López Jiménez, and J.J. Mora Rodríguez, (2009). A comparison among turbulence modelling approaches in the simulation of a square dead zone. XXXIII IAHR Congress, Vancouver, Canada, (2009).

9. C. Gualtieri, P.A. López Jiménez, and J.J. Mora Rodríguez, (2010). Modelling turbulence and solute transport in a square cavity. First European IAHR Congress. Edimburgo, 2010.

10. K. Hanjalić, Closure models for incompressible turbulent flows, Lecture Notes at Von Kármán Institute, pp.75, 2004.

11. D. Infield, U. Eicker, V. Fux, L. Mei, Schumacher, A simplified approach to thermal performance calculation for building integrated mechanically ventilated PV facades. J. Building and Environment. N 41. Pp. 893-901. 2006.

12. G.Kokogiannakis; P. Strachan, Modelling of double ventilated façades according to the CEN Standard 13790 method and detailed simulation. 547 2nd PALENC Conference and 28th AIVC Conference on Building Low Energy Cooling and Advanced Ventilation Technologies in the 21st Century. Crete island, Greece. 2007.

13. BE Launder, Spalding DB. Lectures in mathematical models of turbulence. New York: Academic, 1972

14. P.F. Linden, 1999. The fluid mechanics of natural ventilation. Ann. Rev. Fluid Mech. 31, 201-238.

15. H. Manz, Numerical simulation of heat transfer by natural convection in cavities on façade elements. Energy and Buildings, Vol 35, N 3. Pp 305311. 2003.

16. S. Sarkar, and L. Balakrishnan "Application of a Reynolds-stress turbulence model to the compressible shear layer", ICASE Report 90-18, NASA CR 182002. 1990.

17. C.G. Speziale, S. Sarkar, and T.B. Gatski, 1991. "Modelling the pressurestrain correlation of turbulence: an invariant dynamical systems approach", J. Fluid Mech., 227, pp. 245-272.

18. STAR-CCM+3.04.009, User's Guide, CD-Adapco, USA, 2010.

19. H. Tennekes, JL. Lumley (1972). A first course in turbulence. Cambridge. MA: The MIT Press.

20. M. Todorovic, O. Ecim, A. Marjanovic and I. Randjelovic: (2007) "Natural and Mixed Ventilation Design via CFD and Architectural Modelling", International Journal of Ventilation, 5, (4), 447-458.

21. FM. White. Viscous fluid flow. New York: McGraw-Hill, Inc., 1991.

22. D.C. Wilcox, Turbulence modeling for CFD, DCW Industries, 2002.

23. Z. Yilmaz, Evaluation of energy efficient design strategies for different climatic zones: Comparison of thermal performance of buildings in temperate-humid and hot-dry climate. Energy and Buildings, Vol 39, N 3. Pp 306-316. 2007

Recibido: $17 / 06 / 2010$ Aceptado: 21/03/2011 